\title{
1D Filter for Ring Artifact Suppression
}

DOI:

10.1109/LSP.2016.2554363

\section{Document Version}

Accepted author manuscript

Link to publication record in Manchester Research Explorer

\section{Citation for published version (APA):}

Titarenko, V. (2016). 1D Filter for Ring Artifact Suppression. IEEE Signal Processing Letters, 23(6), 800-804. https://doi.org/10.1109/LSP.2016.2554363

\section{Published in:}

IEEE Signal Processing Letters

\section{Citing this paper}

Please note that where the full-text provided on Manchester Research Explorer is the Author Accepted Manuscript or Proof version this may differ from the final Published version. If citing, it is advised that you check and use the publisher's definitive version.

\section{General rights}

Copyright and moral rights for the publications made accessible in the Research Explorer are retained by the authors and/or other copyright owners and it is a condition of accessing publications that users recognise and abide by the legal requirements associated with these rights.

\section{Takedown policy}

If you believe that this document breaches copyright please refer to the University of Manchester's Takedown Procedures [http://man.ac.uk/04Y6Bo] or contact uml.scholarlycommunications@manchester.ac.uk providing relevant details, so we can investigate your claim.

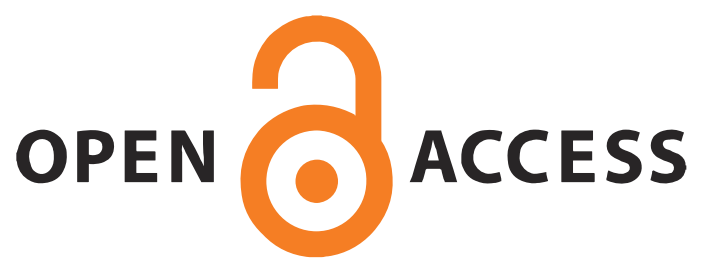




\title{
1D Filter for Ring Artifact Suppression
}

\author{
Valeriy Titarenko
}

\begin{abstract}
Reconstruction algorithms in x-ray tomography provide a user with a set of images. For many scientific problems these images should be segmented. Ring artifacts are the most noticeable artifacts which do not allow a scientist to achieve acceptable segmentation especially in case of noisy input data. Several ring artifact suppression methods based on theory of ill-posed problems exist. In this letter the basic ring artifact suppression algorithm is rewritten as a 1D filter, so it does not require separate data processing and can be combined with standard filtering used in filtered backprojection algorithms.
\end{abstract}

Index Terms-Ring artifacts, Tikhonov regularization, filter, convolution, $x$-ray tomography

\section{INTRODUCTION}

$\mathbf{X}$ -ray tomography is a non-destructive procedure to see an internal structure of a sample. A lot of applications of this technique exist in biology, medicine, engineering, earth, environmental and material sciences. In some areas, e.g. in cancer research, a user is mostly interested in small variations of attenuation coefficients, while other problems require proper segmentation, so further mathematical simulations can provide information of physical/chemical properties of a specimen. The second group of problems can be very sensitive with respect to errors during segmentation process. While x-ray tomography as an experimental technique is prone to various artifacts (limited number of projections, noise, beam hardening, motion), the most critical artifacts for segmentation are ring artifacts. In this letter we consider suppression of ring artifacts for data from synchrotron radiation facilities and laboratory based x-ray imagers used for material science applications.

A standard data collection includes acquisition of projections $I_{\text {proj }}$ of an object rotating in an x-ray beam and a set of flat/white $I_{\text {flat }}$ and dark $I_{\text {dark }}$ field images when there is no specimen and the beam is switched on and off respectively. Thus an optical path along an X-ray through the object can be found as

$$
P=\ln \left(\frac{I_{\text {flat }}-I_{\text {dark }}}{I_{\text {proj }}-I_{\text {dark }}}\right)
$$

and a $2 \mathrm{D} / 3 \mathrm{D}$ reconstruction algorithm is then used to find the internal structure. Due to various instabilities in the $\mathrm{x}$ ray beam [1], [2], defects in a scintillator, optical system or image-recording sensor, the optical path images obtained by applying formula (1) may still have additional systematic peaks. A flat field image is shown in Fig. 1: hard-to remove dirt on various optical surfaces causes decrease in intensity of the beam (darker areas), while scratches and defects on a scintillator increase it (white areas). Considering only one

V. Titarenko is with Henry Moseley X-ray Imaging Facility, The University of Manchester, Manchester, M13 9PL, UK e-mail: valeriy.titarenko@manchester.ac.uk.

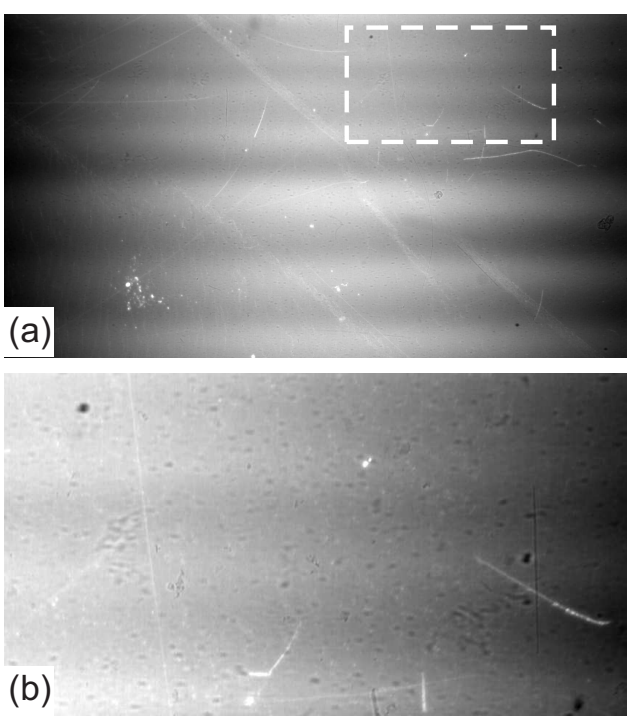

Fig. 1. An example of flat field image acquired at beamline I13, Diamond Light Source: (a) original and (b) zoomed-in areas.

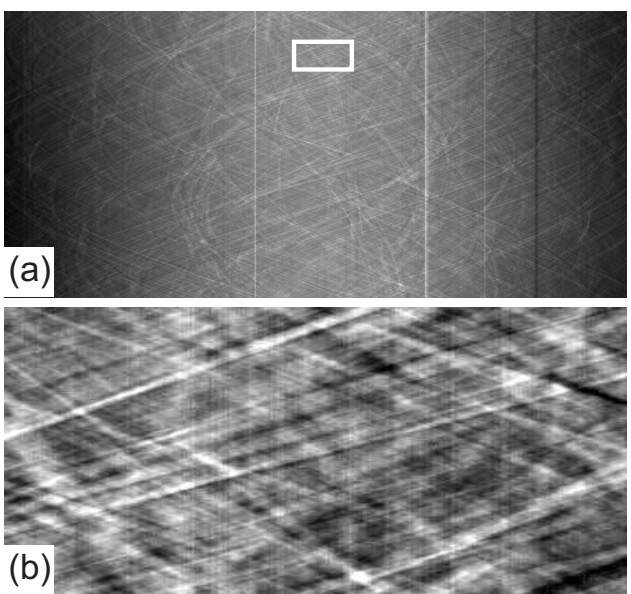

Fig. 2. A example of sinogram for a graphite sample: (a) original and (b) zoomed-in and high contrast areas.

row of a sensor (usually perpendicular to the sample's axis of rotation), the cross-section slice, a so-called sinogram, has lines of higher/lower values caused by theses peaks, see Fig. 2. After reconstruction the slice will contain extra circular structures called ring artifacts. In case of half-rotation scans or cone-beam geometry, these artifacts look like arches of different strengths.

Several groups of methods have already been developed to suppress ring artifacts. Some of them are based on "hardware" solution, for instance in [3] the camera is moving during the scan, so each pixel in the recorded image is made up from contributions from all detector elements. In other methods 
various filters are applied to a sinogram or a reconstructed slice taking into account a linear structure of artifacts on sinogram images or their circular structure on reconstructed images. Details of these approaches can be found in [2], [4]-[21].

In this letter we propose an approach to modify a group of ring artifact suppression methods based on the core results obtained in [7]. These methods described in [2], [8], [10], [11] are implemented as routine procedures in a reconstruction software used at imaging beamlines $\mathrm{I} 12$ and I13 of Diamond Light Source [22], [23], have also applied to data from beamline 2-BM of Advanced Photon Source, Argonne National Laboratory and have already proved their quality and robustness. The aim of the new algorithm is to increase algorithms' performance by reducing amount of data required to be used during ring artifact suppression and combining them with other filters built in standard reconstruction algorithms.

\section{ORIGINAL METHOD}

The idea behind the results from [7] is the following. If there are no artifacts, then two neighboring pixels of a sensor should have similar values. In case of a regular ring artifact it can be described as a constant but unknown value added to the ideal value of the optical path. This unknown value is the same for all projections. As the result the original problem can be written as a problem to minimize a so-called Tiknonov functional [24]

$$
M^{\alpha}=\frac{1}{2} \sum_{k=1}^{n-1}\left(p_{k}-p_{k+1}\right)^{2}+\frac{\alpha}{2} \sum_{k=1}^{n}\left(r_{k}-p_{k}\right)^{2},
$$

where $r_{k}$ is the average recorded optical path for the $k$-th pixel, $p_{k}$ is the unknown optical path, $\alpha>0$ is a regularization parameter and should in principle depend on level of noise in input data. The first term in (2) means $p_{k}$ should not vary much from $r_{k}$, the second term implies restrictions on neighboring values of $p \equiv\left\{p_{k}\right\}_{1}^{n}$. For a given $\alpha$ a solution of (2) exists, is unique and can be found by solving the system of $n$ linear equations

$$
\frac{\partial M^{\alpha}}{\partial z_{k}}=\sum_{l=1}^{n} A_{k l} p_{l}-r_{k}=0
$$

where matrix $A$ is three-diagonal and defined as

$$
\left(\begin{array}{cccccc}
1+\alpha & -1 & 0 & \ldots & 0 & 0 \\
-1 & 2+\alpha & -1 & \ldots & 0 & 0 \\
0 & -1 & 2+\alpha & \ldots & 0 & 0 \\
\vdots & \vdots & \vdots & \ddots & \vdots & \vdots \\
0 & 0 & 0 & -1 & 2+\alpha & -1 \\
0 & 0 & 0 & 0 & -1 & 1+\alpha
\end{array}\right)
$$

In [7] the inverse matrix $A^{-1}$ is found, so the vector $p$ can be found from the vector $r \equiv\left\{r_{k}\right\}_{1}^{n}$. However for practical reasons it is better to have a filter $G \equiv\left\{G_{k}\right\}_{1}^{n}$ such that the solution $p$ is just the convolution $G * r$. Therefore instead of $(n \times n)$-matrix $A^{-1}$ we need to store $n$-vector $G$ and the convolution can be performed by Fast Fourier Transform algorithms.

\section{FILTER}

We intend to find the value of the central element of vector $p$, therefore we suppose $n$ to be an odd number. Let $(n \times n)$ matrix $B_{n}$ be defined as

$$
B_{n}=\left(\begin{array}{cccccc}
2 \sigma-1 & -1 & 0 & \cdots & 0 & 0 \\
-1 & 2 \sigma & -1 & \cdots & 0 & 0 \\
0 & -1 & 2 \sigma & \cdots & 0 & 0 \\
\vdots & \vdots & \vdots & \ddots & \vdots & \vdots \\
0 & 0 & 0 & \cdots & 2 \sigma & -1 \\
0 & 0 & 0 & \cdots & -1 & 2 \sigma-1
\end{array}\right) .
$$

Then $A=B_{n}$ if $\sigma=\alpha / 2+1$. Let us replace the element $(1,1)$ of $B_{n}$ by $\chi$ and denote the corresponding matrix as $F_{n}(\chi)$, $F_{n}(2 \sigma-1)=B_{n}$. We use the first row of $F_{n}(\chi)$ to find its determinant, then $\operatorname{det} F_{n}(\chi)=\chi \operatorname{det} F_{n-1}(2 \sigma)-\operatorname{det} F_{n-2}(2 \sigma)$. Considering cases of $\chi=2 \sigma$ and $\chi=2 \sigma-1$ we get $\operatorname{det} B_{n}=$ $\operatorname{det} F_{n}(2 \sigma)-\operatorname{det} F_{n-1}(2 \sigma)$ and $\operatorname{det} F_{n}(2 \sigma)+\operatorname{det} F_{n-2}(2 \sigma)=$ $2 \sigma \operatorname{det} F_{n-1}(2 \sigma)$, so $\operatorname{det} B_{n}+\operatorname{det} B_{n-2}=2 \sigma \operatorname{det} B_{n-1}$. Therefore we need to find a sequence $\left\{b_{n}\right\}$ such that

$$
b_{n}+b_{n-2}=2 \sigma b_{n-1}
$$

and $b_{n}=\operatorname{det} B_{n}$. Note that matrices $B_{n}$ are formally defined for $n \geq 3$. One can find their determinants explicitly for $n=3$ and 4 , so $b_{3}=8 \sigma^{3}-8 \sigma^{2}-2 \sigma+2, b_{4}=16 \sigma^{4}-16 \sigma^{3}-$ $8 \sigma^{2}+8 \sigma$ and using (6) we extrapolate values $b_{n}$ for $n<3$ : $b_{2}=4 \sigma^{2}-4 \sigma, b_{1}=2 \sigma-2, b_{0}=0$.

If $\sigma \in[-1,1]$ and we set $b_{n}=\tau \sin (n \omega+\psi)$ and $\sigma=\cos \omega$, then (6) is met. Taking into account $b_{0}$ and $b_{1}$ we get $\psi=0$ and

$$
\begin{gathered}
\tau=\frac{2 \sigma-2}{\sin \omega}=\frac{2(\cos \omega-1)}{\sin \omega}, \\
\operatorname{det} B_{n}=2(\cos \omega-1) \frac{\sin n \omega}{\sin \omega} .
\end{gathered}
$$

Matrix $B_{n}$ is an $(n \times n)$-matrix, so $\operatorname{det} B_{n}$ can not have more than $n$ roots. Below we show that there are $n$ roots for $\sigma \in$ $[-1,1]$, thus there is no need to consider cases $|\sigma|>1$.

The first root can be found from $\cos \omega=1$. The other roots can be found from $\sin (n \omega)=0$, so $n \arccos \sigma=k \pi$ and $\sigma=\cos (k \pi / n), k=0, \ldots, n-1$. All eigenvalues of matrix $B_{n}$ can be written as $\lambda_{k}=2\left(\sigma+\rho_{k}\right)$, where $\rho_{k}=\cos (k \pi / n)$, $k=0, \ldots, n-1$.

Denote $\theta=k \pi / n$, then for a given eigenvalue $\lambda=2(\sigma+$ $\left.\rho_{k}\right)$ the corresponding eigenvector $y_{q}, q=1, \ldots, n$ :

$$
y_{q}=(-1)^{q-1}\left[1+2 \sum_{s=1}^{q-1} \cos (s \theta)\right] \text {. }
$$

In [25] the following formula is given

$$
\sum_{s=0}^{N-1} \cos (a+s b)=\cos \left(a+\frac{N-1}{2} b\right) \frac{\sin N b / 2}{\sin b / 2},
$$

thus if set $a=0$, then

$$
y_{q}=(-1)^{q-1} \frac{\sin (2 q-1) \theta / 2}{\sin \theta / 2} .
$$


Now we normalize vector $y$ and therefore rewrite $\sin ^{2} \frac{2 q-1}{2} \theta$ as $(1-\cos (2 q-1) \theta) / 2$. Substituting $a=\theta$ and $b=2 \theta$ into (10) we find

$$
\begin{gathered}
\sum_{q=1}^{n} \cos (2 q-1) \theta=\sum_{s=0}^{n-1} \cos (\theta+2 s \theta)=\frac{\sin 2 n \theta}{2 \sin \theta} \\
\sum_{q=1}^{n} \sin ^{2} \frac{2 q-1}{2} \theta=\frac{1}{4}\left(2 n-\frac{\sin 2 n \theta}{\sin \theta}\right) .
\end{gathered}
$$

Since $\theta=k \pi / n$, then $\sin 2 n \theta=\sin 2 \pi k=0$ for any integer $k$. Note that $\sin \theta \neq 0$ for $k=1, \ldots, n-1$ and $\sin \theta=0$ for $k=n$. In the last case we get $y_{q}=1$, so its normalization is trivial. Finally, we get the normalized vector $y$ with elements

$$
y_{q}= \begin{cases}\frac{(-1)^{q-1}}{\sqrt{n / 2}} \sin \frac{(2 q-1) k \pi}{2 n}, & k=1, \ldots, n-1, \\ \frac{1}{\sqrt{n}}, & k=n .\end{cases}
$$

The original matrix $B=B_{n}$ from (5) can be written as

$$
B=Y D Y^{T},
$$

where $Y$ is an orthogonal matrix, i.e. $Y^{-1}=Y^{T}$, with elements

$$
Y_{i j}= \begin{cases}\frac{(-1)^{i-1}}{\sqrt{n / 2}} \sin \frac{(2 i-1) j \pi}{2 n}, & j=1, \ldots, n-1, \\ \frac{1}{\sqrt{n}}, & j=n,\end{cases}
$$

$D$ is a diagonal matrix with elements $D_{i i}=$ $2(\sigma+\cos (i \pi / n))$, see [26].

The inverse of matrix $B_{n}$ can then be written as

$$
B^{-1}=Y D^{-1} Y^{T},
$$

where $D^{-1}$ is a diagonal matrix with elements $D_{i i}^{-1}=$ $1 /[2(\sigma+\cos (i \pi / n))]$. Therefore

$$
\left.D^{-1} Y^{T}\right|_{k j}=\sum_{q=1}^{n} D_{k q}^{-1} Y_{q j}^{T}=D_{k k}^{-1} Y_{k j}^{T}=D_{k k}^{-1} Y_{j k}
$$

and the elements

$$
B_{i j}^{-1}=\sum_{k=1}^{n} Y_{i k} D_{k k}^{-1} Y_{j k}
$$

of $B^{-1}$ can be written as

$B_{i j}^{-1}=\frac{1}{n}\left[\sum_{k=1}^{n-1} \frac{(-1)^{i+j}}{\sigma+\cos \frac{k \pi}{n}} \sin \frac{(2 i-1) k \pi}{2 n} \sin \frac{(2 j-1) k \pi}{2 n}+\frac{1}{2(\sigma-1)}\right]$.

Taking in to account that

$$
\sin \frac{(2 i-1)(2 n-k) \pi}{2 n}=\sin \frac{(2 i-1) k \pi}{2 n}
$$

and $\cos \frac{n \pi}{n}=-1$ we get

$$
B_{i j}^{-1}=\frac{1}{2 n} \sum_{k=1}^{2 n-1} \frac{(-1)^{i+j}}{\sigma+\cos \frac{k \pi}{n}} \sin \frac{(2 i-1) k \pi}{2 n} \sin \frac{(2 j-1) k \pi}{2 n} .
$$

All matrices mentioned above have odd width/height and indexes starting from 1. Our aim is to find a filter, so it is more convenient to rewrite matrices/vectors as ones centered with respect to the central element. If a standard matrix $Q$ is given, then by $\check{Q}$ we denote the centred one. So instead of matrix $B_{i j}^{-1}$ defined for $i=1, \ldots, n, j=1, \ldots, n$ we subtract $n$ from $k$ and $(\check{n}+1)$, where $\check{n}=(n-1) / 2$, from the other indexes of the above formula and get

$$
\begin{aligned}
\check{B}_{i j}^{-1} & =\frac{1}{2 n} \sum_{k=-(n-1)}^{n-1} \frac{(-1)^{i+j}}{\sigma+\cos \frac{(k+n) \pi}{n}} \\
& \times \sin \frac{(2(i+\check{n})+1)(k+n) \pi}{2 n} \sin \frac{(2(j+\check{n})+1)(k+n) \pi}{2 n}, \\
& =\frac{1}{4 n} \sum_{k=-(n-1)}^{n-1} \frac{\cos \frac{(i-j) k \pi}{n}+\cos \frac{(i+j+n) k \pi}{n}}{\sigma-\cos \frac{k \pi}{n}} .
\end{aligned}
$$

For the central row of $\check{B}^{-1}$ we get

$$
\check{B}_{0 j}^{-1}=\frac{1}{4 n} \sum_{k=-(n-1)}^{n-1} \frac{\cos \frac{j k \pi}{n}\left(1+(-1)^{k}\right)}{\sigma-\cos \frac{k \pi}{n}} .
$$

For odd $k$ the value of $\left(1+(-1)^{k}\right)=0$, so we need to consider only even $k$ and use $s=k / 2$ :

$$
\check{B}_{0 j}^{-1}=\frac{1}{2 n} \sum_{s=-\check{n}}^{\check{n}} \frac{\cos \frac{2 j s \pi}{n}}{\sigma-\cos \frac{2 s \pi}{n}} .
$$

For large values of $n$ we may replace the sum by the integral

$$
\frac{1}{2 n} \int_{-n / 2}^{n / 2} \frac{\cos \frac{2 j s \pi}{n}}{\sigma-\cos \frac{2 s \pi}{n}} d s .
$$

Taking into account $\sigma=\alpha / 2+1$ we get values of filter $\check{G}$

$$
\check{G}_{j}=\frac{2}{(2+\alpha) \pi} \int_{0}^{\pi} \frac{\cos j \xi}{1-\frac{2}{2+\alpha} \cos \xi} d \xi .
$$

Let us denote

$$
\begin{gathered}
\sin \phi=\frac{2}{2+\alpha}, \quad \cos \phi=\frac{\sqrt{\alpha(4+\alpha)}}{2+\alpha}, \\
I_{m} \equiv \int_{0}^{\pi} \frac{\cos m x}{1-\cos x \sin \phi} d x
\end{gathered}
$$

$\phi \in\left(0, \frac{\pi}{2}\right)$ since $\alpha>0$. Using the formula $\cos m x+\cos (m-$ 2) $x=2 \cos x \cos (m-1) x$ we obtain for $m>1$

$$
\begin{aligned}
I_{m} & +I_{m-2}=\int_{0}^{\pi} \frac{2 \cos x \cos (m-1) x}{1-\cos x \sin \phi} d x \\
& =\frac{2}{\sin \phi} \int_{0}^{\pi}\left(\frac{1}{1-\cos x \sin \phi}-1\right) \cos (m-1) x d x \\
& =\frac{2}{\sin \phi} I_{m-1},
\end{aligned}
$$

since $\int_{0}^{\pi} \cos (m-1) x d x=0$. So we get the recurrent formula

$$
I_{m}=\frac{2}{\sin \phi} I_{m-1}-I_{m-2} \text {. }
$$

We may explicitly find that

$$
I_{0}=\frac{\pi}{\cos \phi}, \quad I_{1}=\frac{\pi}{\cos \phi} \frac{\sin \phi}{1+\cos \phi}
$$

and check that $I_{m}$ is a geometric sequence, i.e. $I_{m+1}=\gamma I_{m}$, where $\gamma=\sin \phi /(1+\cos \phi)$. So the values of filter $\check{G}$ can be written as

$$
\check{G}_{j}=\frac{2}{\sqrt{\alpha(4+\alpha)}}\left(\frac{2+\alpha-\sqrt{\alpha(4+\alpha)}}{2}\right)^{|j|} .
$$




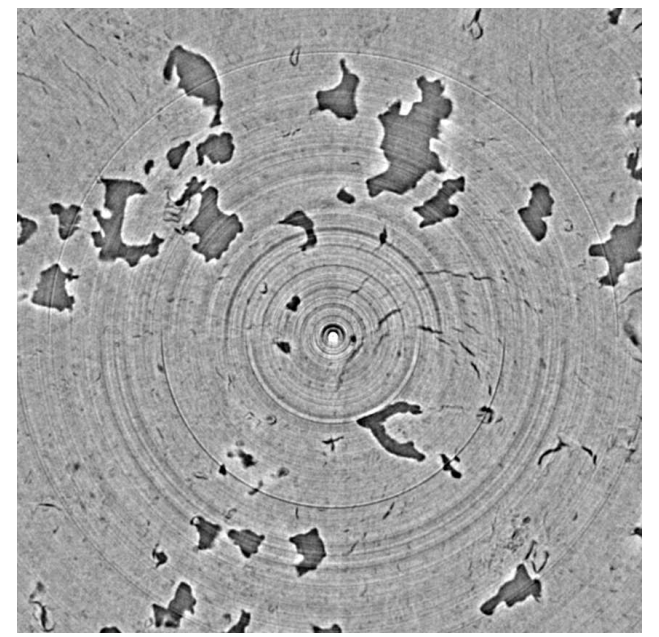

Fig. 3. A horizontal slice of the graphite after the standard flat-field correction procedure is applied.

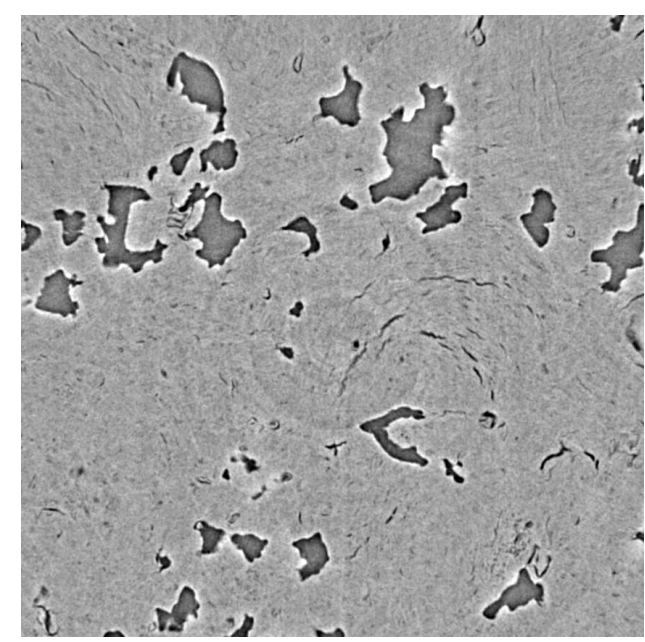

Fig. 4. The same slice reconstructed after the ring artifact suppression method is applied, $\alpha=500$.

It is easy to check that $\gamma \in(0,1)$, the sequence is a decreasing one, i.e. $\check{G}_{|j+1|}<\check{G}_{|j|}$, and $\sum_{j=-\infty}^{\infty} \check{G}_{j}=1$.

Above the classical approach used in the theory of inverse and ill-posed problems is used. In this case values of regularization parameter $\alpha$ belong to the positive half-line, i.e. $\alpha \in(0,+\infty)$. It may be more convenient to normalize the range. Let us introduce parameter $\beta=1-\hat{G}_{0}$, then

$$
\begin{gathered}
\alpha=\frac{2\left(\sqrt{(1-\beta)^{2}+1}-(1-\beta)\right)}{1-\beta}, \\
\check{G}_{j}=(1-\beta)\left(\frac{\sqrt{(1-\beta)^{2}+1}-1}{1-\beta}\right)^{|j|} .
\end{gathered}
$$

So $\beta \in[0,1), \beta=0$ corresponds to the case of no filtering, i.e. $\check{G}_{0}=1$ and $\check{G}_{j}=0$ for $j \neq 0$. The value of $\beta$ cannot reach 1 but in case of approaching to it the filter tends to be the mean filter.

\section{DISCUSSION}

The formula (29) proposed in this letter is applied to suppress ring artifacts for a graphite sample. The data was acquired at beamline I13 of Diamond Light Source, UK [23]. There were $4001 \mathrm{x}$-ray projections acquired, each projection was a 16-bit $2560 \times 2160$ image. As the beamline provides users with almost parallel geometry of the x-ray beam, reconstruction of one horizontal section of a volume requires only one sinogram, i.e. one data from one row of a sensor. For a given slice the standard flat-field correction procedure defined in (1) is applied. In Figure 3 one can see that ring artifacts have regular structure, so their strengths are almost the same along half-circles. The reconstruction based on the filter (29) for $\alpha=500$ is performed, see Figure 4 .

Of course, the choice of parameter $\alpha$ should depend on level on noise/strength of ring artifacts. The smaller parameter $\alpha$ is, the closer the slice is to the one after the standard flatfield correction, i.e. to Figure 3. If the value of $\alpha$ is too high, then additional wave structures may appear on the image. See examples in [27]. For ring artifacts dependant on angular position of a specimen the method presented in [11] can be used.

Standard fast reconstruction algorithms like filtered backprojection (FBP) or Feldkamp-Davis-Kress (FDK) for parallel and cone geometry of the X-ray beam consist of two steps: filtering and backprojection [28]. The algorithm presented in this letter is designed to be performed before reconstruction. Having it written in the form of filtering operation allows us to combine preprocessing and reconstruction steps by using the modified filter for FBP and FDK which can be defined as the convolution of the ring artefact filter with the standard one.

\section{ACKNOWLEDGMENT}

The author thank Dr Zhanna Mileeva for the raw data from beamline I13 of Diamond Light Source.

\section{REFERENCES}

[1] J. Hinebaugh, P. R. Challa, and A. Bazylak, "Accounting for lowfrequency synchrotron X-ray beam position fluctuations for dynamic visualizations," Journal of Synchrotron Radiation, vol. 19, no. 6, pp. 994-1000, Nov 2012.

[2] V. Titarenko, S. Titarenko, P. J. Withers, F. De Carlo, and X. Xiao, "Improved tomographic reconstructions using adaptive time-dependent intensity normalization," Journal of Synchrotron Radiation, vol. 17, no. 5, pp. 689-699, 2010.

[3] G. R. Davis, A. N. Z. Evershed, and D. Mills, "Quantitative high contrast X-ray microtomography for dental research," Journal of Dentistry, vol. 41, no. 5, pp. 475-482, 2013.

[4] J. Sijbers and A. Postnov, "Reduction of ring artefacts in high resolution micro-CT reconstructions," Physics in Medicine and Biology, vol. 49, no. 14, pp. N247-N253, 2004.

[5] B. Münch, P. Trtik, F. Marone, and M. Stampanoni, "Stripe and ring artifact removal with combined wavelet - Fourier filtering," Optics Express, vol. 17, no. 10, pp. 8567-8591, 2009.

[6] D. Prell, Y. Kyriakou, and W. A. Kalender, "Comparison of ring artifact correction methods for flat-detector CT," Physics in Medicine and Biology, vol. 54, no. 12, pp. 3881-3895, 2009.

[7] S. Titarenko, P. J. Withers, and A. Yagola, "An analytical formula for ring artefact suppression in X-ray tomography," Applied Mathematics Letters, vol. 23, no. 12, pp. 1489-1495, 2010.

[8] S. Titarenko, V. Titarenko, A. Kyrieleis, and P. Withers, "A ring artifact suppression algorithm based on a priori information," Applied Physics Letters, vol. 95, no. 7, p. 071113, 2009. 
[9] F. Sadi, S. Y. Lee, and M. K. Hasan, "Removal of ring artifacts in computed tomographic imaging using iterative center weighted median filter," Computers in Biology and Medicine, vol. 40, no. 1, pp. 109-118, 2010.

[10] V. Titarenko, R. Bradley, C. Martin, P. J. Withers, and S. Titarenko, "Regularization methods for inverse problems in X-ray tomography," in Developments in X-Ray Tomography VII, vol. 7804, 2010.

[11] S. Titarenko, V. Titarenko, A. Kyrieleis, P. J. Withers, and F. De Carlo, "Suppression of ring artefacts when tomographing anisotropically attenuating samples," Journal of Synchrotron Radiation, vol. 18, no. 3, pp. 427-435, 2011.

[12] S. Rashid, S. Y. Lee, and M. K. Hasan, "An improved method for the removal of ring artifacts in high resolution CT imaging," Eurasip Journal on Advances in Signal Processing, vol. 2012, no. 1, 2012.

[13] Z. Wei, S. Wiebe, and D. Chapman, "Ring artifacts removal from synchrotron CT image slices," Journal of Instrumentation, vol. 8, no. 6, 2013.

[14] F. Brun, A. Accardo, G. Kourousias, D. Dreossi, and R. Pugliese, "Effective implementation of ring artifacts removal filters for synchrotron radiation microtomographic images," in International Symposium on Image and Signal Processing and Analysis, ISPA, 2013, pp. 672-676.

[15] E. X. Miqueles, J. Rinkel, F. O’Dowd, and J. S. V. Bermúdez, "Generalized Titarenko's algorithm for ring artefacts reduction," Journal of Synchrotron Radiation, vol. 21, no. 6, pp. 1333-1346, 2014.

[16] C. Altunbas, C.-J. Lai, Y. Zhong, and C. C. Shaw, "Reduction of ring artifacts in CBCT: Detection and correction of pixel gain variations in flat panel detectors," Medical Physics, vol. 41, no. 9, 2014.

[17] D. Jha, H. O. Sørensen, S. Dobberschütz, R. Feidenhans'L, and S. L. S. Stipp, "Adaptive center determination for effective suppression of ring artifacts in tomography images," Applied Physics Letters, vol. 105, no. 14, 2014.

[18] P. Paleo and A. Mirone, "Ring artifacts correction in compressed sensing tomographic reconstruction," Journal of Synchrotron Radiation, vol. 22, pp. 1268-1278, 2015.

[19] B. Mao, D. Xiao, X. Xiong, X. Chen, W. Zhang, and Y. Kang, “Accelerating ring artifacts reduction for cone beam CT based on projection data using CUDA," in Medical Imaging Physics and Engineering (ICMIPE), 2013 IEEE International Conference on, Oct 2013, pp. 30-33.

[20] D. Zeng, J. Ma, Y. Zhang, Z. Bian, J. Huang, and W. Chen, "An improved ring artifact removal approach for flat-panel detector based computed tomography images," in Nuclear Science Symposium and Medical Imaging Conference (NSS/MIC), 2013 IEEE, Oct 2013, pp. $1-4$.

[21] Y.-W. Chen, G. Duan, A. Fujita, K. Hirooka, and Y. Ueno, "Ring artifacts reduction in cone-beam CT images based on independent component analysis," in Instrumentation and Measurement Technology Conference, 2009. I2MTC 'O9. IEEE, May 2009, pp. 1734-1737.

[22] M. Drakopoulos, T. Connolley, C. Reinhard, R. Atwood, O. Magdysyuk, N. Vo, M. Hart, L. Connor, B. Humphreys, G. Howell, S. Davies, T. Hill, G. Wilkin, U. Pedersen, A. Foster, N. De Maio, M. Basham, F. Yuan, and K. Wanelik, "I12: The joint engineering, environment and processing (JEEP) beamline at diamond light source," Journal of Synchrotron Radiation, vol. 22, pp. 828-838, 2015.

[23] Z. D. Pešić, A. D. Fanis, U. Wagner, and C. Rau, "Experimental stations at I13 beamline at Diamond Light Source," Journal of Physics: Conference Series, vol. 425, no. 18, p. 182003, 2013.

[24] A. N. Tikhonov, A. V. Goncharsky, V. V. Stepanov, and A. G. Yagola, Numerical Methods for the Solution of Ill-Posed Problems, ser. Mathematics and Its Applications. Netherlands: Springer, 1995, vol. 328.

[25] I. S. Gradshteyn and I. M. Ryzhik, Table of integrals, series, and products. Amsterdam: Elsevier/Academic Press, 2015.

[26] N. Higham, Functions of Matrices. Society for Industrial and Applied Mathematics, 2008. [Online]. Available: http://epubs.siam.org/doi/abs/10.1137/1.9780898717778

[27] S. Titarenko, V. Titarenko, A. Kyrieleis, and P. J. Withers, "A priori information in a regularized sinogram-based method for removing ring artefacts in tomography," Journal of Synchrotron Radiation, vol. 17, no. 4, pp. 540-549, Jul 2010.

[28] F. Natterer and F. Wübbeling, Mathematical Methods in Image Reconstruction. Society for Industrial and Applied Mathematics, 2001. 